\title{
Experimental and Numerical Study of Twin Circular Water Jets Impinging on a Moving Thin Film
}

\author{
Hitoshi FUJIMOTO, ${ }^{*}$ Yasuyuki LEE, Ryosuke KATO, Takayuki HAMA and Hirohiko TAKUDA \\ Graduate School of Energy Science, Kyoto University, Kyoto, 606-8501 Japan. \\ (Received on February 15, 2013; accepted on April 18, 2013)
}

\begin{abstract}
In this study, we investigated the flow characteristics of two circular water jets impinging on a moving surface covered with a water film as fundamental research on strip cooling. Experiments and numerical simulations were conducted under non-heated surface conditions. The experiments were recorded on video. The jet velocity, nozzle-to-plate distance, nozzle-to-nozzle spacing, and flow rates of the water film were varied systematically. Depending on the flow conditions, three types of flows were found to exist between the two modes: stable, unstable, and transient. We propose a simple theoretical model for predicting the critical boundary at which the flow is in the "stable mode." In the numerical simulation, the Navier-Stokes equation system for a three-dimensional incompressible unstable viscous fluid was solved using a finite difference method. The effects of viscosity, gravity, and presence of a free liquid surface with surface tension were considered. The flow characteristics of the "unstable mode" are discussed in detail to offer a better understanding of its physics.
\end{abstract}

KEY WORDS: strip cooling; impinging jet; free surface flow; numerical simulation; hydraulic jump.

\section{Introduction}

Impinging liquid jets are widely used in steel-making plants. In hot-rolling, hot steel sheets are passed through the finishing mill and rapidly cooled on a run-out-table (ROT) by arrays of circular water jets from the final rolling temperature to the coiling temperature. Because precise temperature control of steel sheets is essential to making high-quality products, the heat transfer characteristics of this cooling process have been studied extensively through both experiments $^{1-4)}$ and numerical simulations. ${ }^{5-8)}$

In ROT cooling, a large amount of water impinges upon a moving steel sheet to form a thick water film over the solid sheet. Therefore, most of the circular water jets in an array impinge not on the dry hot steel sheet but on the pre-existing liquid film. The water jet-liquid film flow interaction is very complex by nature. Although the impingement of a liquid jet on a moving liquid film is a basic component of ROT cooling, we know little about the associated flow mechanics.

In widely used models for predicting the temperature history of hot steel in strip cooling, only the heat conduction equation is solved with boundary conditions at solid surfaces, where time- and space-averaged heat transfer coefficients are imposed. The flow structure of the cooling fluid has a large impact on the local heat transfer characteristics between the cooling fluid and the solid, but its effect is insufficiently reflected by the empirical heat transfer coefficients. Understanding the physics of the cooling fluid is

\footnotetext{
* Corresponding author: E-mail: h-fujimoto@energy.kyoto-u.ac.jp DOI: http://dx.doi.org/10.2355/isijinternational.53.1427
}

indispensable to accurately predicting the heat transfer process involved.

In light of the above background, we previously studied the impingement of single water jets on a thin water film flow. ${ }^{9)}$ The flows were investigated using videography and three-dimensional (3D) numerical simulations under the condition of a non-heated solid. High-speed videography revealed that depending on the experimental conditions, three types of flow exist between the two modes: stable, unstable, and transient. In addition, numerical simulations were used to explore the mechanism underlying the unsteady flow motion in detail. Because the previous study dealt with single-jet impingement, in the present study, we addressed the characteristics of multiple impinging jets, which have not been clarified so far. Although multiple-jet impingement on a solid surface has been studied by other researchers, ${ }^{10,11)}$ detailed knowledge on the associated flow mechanics is still lacking.

The objective of this study was to investigate the flow characteristics of two circular water jets impinging on a moving surface covered with a water film under non-heated conditions, as shown in Fig. 1(a). Experiments and numerical simulations were conducted. The experiments were captured using a video camera. The jet velocity, nozzle-to-plate distance, nozzle-to-nozzle spacing, and flow rates of the water film were varied systematically. We found three flow categories similar to those under single-jet impingement that depended on the flow conditions. We then built a simple theoretical model to predict the critical boundary at which the flow is in "stable mode." In the numerical simulation, the Navier-Stokes equation system for 3D incompressible and 


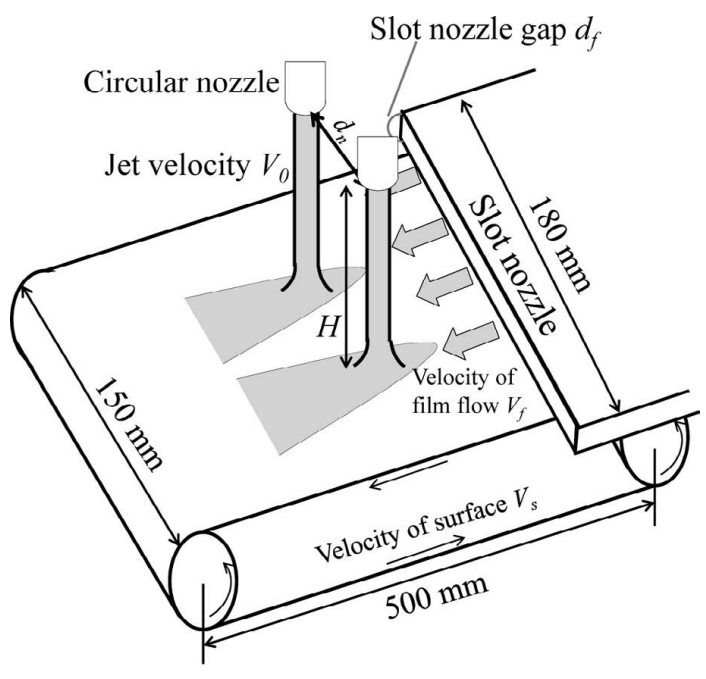

(a)

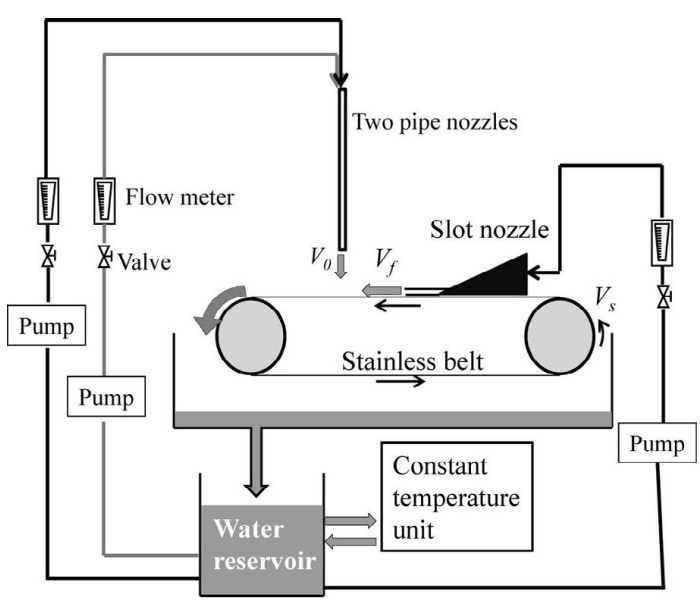

(b)

Fig. 1. Schematics of (a) experimental setup and (b) piping diagram.

unstable viscous fluids was solved using a finite difference method. The effects of viscosity, gravity, and presence of a free liquid surface with surface tension were considered. The flow characteristics of the unstable mode are discussed in detail to offer a better understanding of its physics.

\section{Experimental Setup}

Figure 1 shows (a) a schematic of the experimental setup and (b) the piping diagram. The setup comprises a water reservoir with a constant-temperature unit, three pipelines for making a twinjet and film flow, a moving stainless belt attached to a pair of rolls, and observation equipment. Except for the number of circular jets and width of the moving belt, the setup and measurement procedure in this study were similar to those in our previous work $;{ }^{9)}$ therefore, we provide only a brief explanation here.

Deionized water was used as the test liquid. The water temperature was maintained at $20 \pm 0.1^{\circ} \mathrm{C}$ using a constanttemperature unit. A pair of circular jets was generated using two pipe nozzles having an inner diameter of $7.0 \mathrm{~mm}$ and length of $560 \mathrm{~mm}$. The mean velocity $V_{0}$ at the nozzle exit was varied from 0.4 to $2.0 \mathrm{~m} / \mathrm{s}$. The nozzle-to-moving surface spacing $H$ was set to 20 or $40 \mathrm{~mm}$, and the distance $d_{n}$ between the two nozzle centers was set to 20,30 , or $40 \mathrm{~mm}$.

A 0.15 -mm-thick and $150-\mathrm{mm}$-wide stainless steel belt was used as the moving solid. The moving speed $V_{s}$ of the belt could be varied from $0 \mathrm{~m} / \mathrm{s}$ to $0.9 \mathrm{~m} / \mathrm{s}$. A thin liquid film flow was generated using a slot nozzle unit attached to the moving belt unit. The slot nozzle gap $d_{f}$ was set to $2.3 \mathrm{~mm}$.

Each experiment was conducted such that the preset values of the jet velocity, film flow velocity, and moving belt velocity remained constant. The flow rate of each nozzle was monitored using three flow meters and maintained at the preset value by adjustment of the regulating valves. The water flow rates were measured directly from the volume of water discharged from the nozzle exit during a certain period $(5-60 \mathrm{~s})$. The accuracy of these measurements was $\pm 0.005 \mathrm{l} / \mathrm{s}$. The mean velocities issued from the circular nozzles and slot nozzle were determined using the measured values. In this study, the mean velocity from the slot nozzle was equal to the speed of the belt's motion.

To understand the unstable flow characteristics, the experiments were recorded using high-speed videography. A high-brilliance metal halide lamp was used as the light source. In most experiments, video images were captured at a frame rate of $300 \mathrm{fps}$ with a resolution of $512 \times 384$ pixels.

\section{Numerical Simulation}

\subsection{Conservation Equations}

Figure 2 shows a schematic of the computational domain and the definition of the coordinate system. The $x$ and $y$ directions represent the motional and span-wise directions, respectively, of the solid. The direction normal to the solid is represented by $z$. The origin is set to the midpoint of the distance between the points at which the two jets impinge on the solid surface.

The computational domain has two fluids: water and air. Both fluids are assumed to obey the Navier-Stokes system of equations for incompressible viscous fluids in a 3D Cartesian coordinate system. The effects of gravity, viscosity, and surface tension at the free liquid surface are considered. The flows are assumed to be laminar. The corresponding conservation equations under the assumption of constant thermofluid properties are as follows.

$$
\begin{array}{r}
\frac{\partial u_{i}}{\partial x_{i}}=0 \\
\frac{\partial u_{i}}{\partial t}+u_{j} \frac{\partial u_{i}}{\partial x_{j}}=F_{i}+F_{s v, i}-\frac{1}{\rho} \frac{\partial p}{\partial x_{i}}+\frac{\mu}{\rho} \frac{\partial^{2} u_{i}}{\partial x_{j}^{2}}
\end{array}
$$

where $t, x_{i}$, and $u_{i}$ are the time, coordinates, and velocity, respectively. $p, \rho$, and $\mu$ denote the pressure, apparent fluid density, and apparent kinematic molecular viscosity, respectively. $F_{i}$ denotes the volume force due to gravity, and $F_{s v, i}$ denotes the effect of surface tension.

The time evolution of the free surface was tracked using the volume of fluid/piecewise linear interface calculation (VOF/PLIC) method. ${ }^{12,13)}$ The volume fraction $\phi$ of a liquid in a computational cell is introduced ${ }^{14)}$ here; the equation for determining $\phi$ is as follows:

$$
\frac{\partial \phi}{\partial t}+u_{i} \frac{\partial \phi}{\partial x_{i}}=0 .
$$




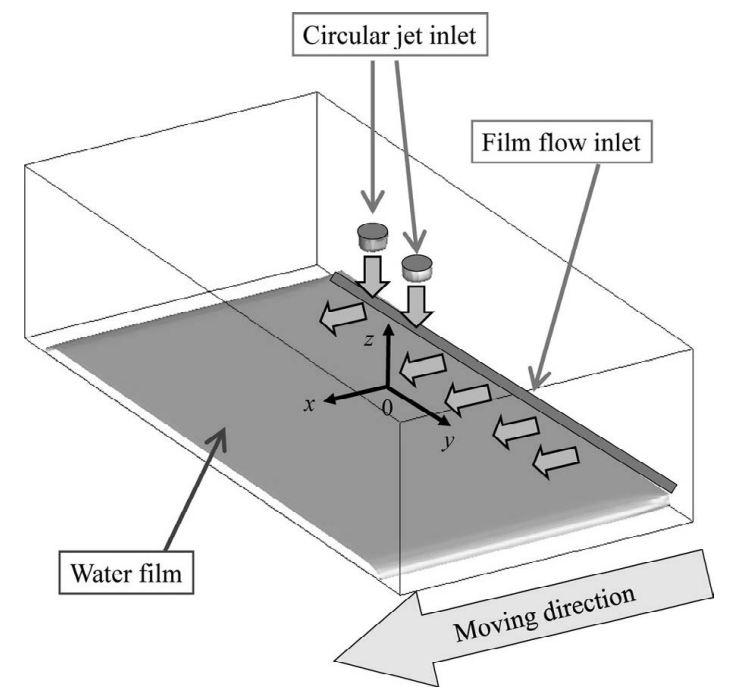

Fig. 2. Schematic of computational domain and coordinate system.

In the VOF/PLIC method, flat planes represent free surfaces in computational cells. The volume fraction is updated using the local velocity and the unit vector normal to the free surface, $\vec{n}$. To calculate the unit normal vector accurately, we used a level-set function $\varphi$ as defined by the distance between a free surface plane and the cell center. Note that the level-set function is given for not only the free surface cell containing two types of fluid but also the single-phase cells near the free surface cells. The unit normal vector can be determined by using the level-set function as follows:

$$
\vec{n}=\nabla \varphi /|\nabla \varphi| \text {. }
$$

Based on the definition of the level-set function, $|\nabla \varphi|=1$ is true. Furthermore, the following evolution equation holds for the level-set function.

$$
\frac{\partial \varphi}{\partial t}+u_{i} \frac{\partial \varphi}{\partial x_{i}}=0
$$

However, the level-set function cannot be calculated accurately using Eq. (5) alone, and a procedure to reconstruct it is needed. In the present study, we numerically solved the following evolution equation to reconstruct the level-set function: ${ }^{15}$

$$
\frac{\partial \varphi}{\partial \tau}+\mathbf{u}_{\varphi} \nabla \varphi=\operatorname{sign}(\varphi), \operatorname{sign}(\varphi)=\frac{\varphi_{0}}{\sqrt{\varphi_{0}{ }^{2}+\delta_{0}^{2}}}, \mathbf{u}_{\varphi}=\operatorname{sign}(\varphi) \frac{\nabla \varphi}{|\nabla \varphi|}
$$

where $\tau, \varphi_{0}$, and $\delta_{0}$ are the pseudo-time, initial level-set function at each time step, and a small value to avoid dividing by zero when calculating $\operatorname{sign}\left(\varphi_{0}\right)$, respectively. The equation was solved until the solution of $\partial \varphi / \partial \tau \sim 0$ was obtained. The following relationship between the level-set function and the volume fraction holds at free surface cells:

$$
\phi=f(\varphi) \text {. }
$$

To calculate the level-set function accurately, we imposed Eq. (7) as a constraining condition for solving Eq. (6).

The level-set function was used to calculate the surface tension force using the continuum surface force (CSF) model $^{16)}$ in the following form:

$$
F_{s v}=\sigma \kappa \nabla \varphi,
$$

where $\sigma$ and $\kappa$ denote the surface tension coefficient and mean curvature of the free surface, respectively. The curvature of the free surface, $\kappa$, is calculated as

$$
\kappa=-(\nabla \cdot \vec{n}) .
$$

The apparent density and viscosity are given using the volume fraction as follows:

$$
\begin{aligned}
& \rho=\rho_{\text {water }} \phi+\rho_{\text {air }}(1-\phi) \\
& \mu=\mu_{\text {water }} \phi+\mu_{\text {air }}(1-\phi) .
\end{aligned}
$$

We now explain the boundary conditions. The no-slip velocity condition was employed at the lower boundary corresponding to the moving plate surface (see also Fig. 2). At the boundaries corresponding to the exits of the circular and slot nozzles, we imposed inflow conditions. The outflow condition was adopted for the other boundaries. This condition allows for the outward flow of liquid through the boundary but prohibits inward flow.

The dimensions of the computational domain were $-0.04 \mathrm{~m}$ $\leq x \leq 0.1 \mathrm{~m},-0.08 \mathrm{~m} \leq y \leq 0.08 \mathrm{~m}$, and $0 \mathrm{~m} \leq z \leq 0.04 \mathrm{~m}$. The domain was divided into $230 \times 260 \times 80$ meshes in the $x, y$, and $z$ directions, respectively. In the $\mathrm{z}$-direction, a very fine mesh was set close to the solid surface, where very thin velocity boundary layers are present. In total, there were 4784000 grid points. The conservation equations were solved using a finite difference method. A fractional step method was adopted to solve the Navier-Stokes equations in accordance with the cubic interpolation program (CIP)combined and unified procedure (CCUP) developed by Yabe et $a l{ }^{17)}$ The advection terms in the Navier-Stokes equations and Eq. (6) for reconstructing the level-set function were solved numerically using the CIP scheme. ${ }^{17)}$ The diffusion terms were approximated with the central difference method and solved using the implicit method. The time increment $\Delta t$ was determined from the following condition to obtain stable numerical results:

$$
\Delta t=0.2 \times \min \left[\frac{\Delta x_{i}}{\left|u_{i}\right|}, \frac{2 \Delta x_{i}}{\sqrt{u_{i}^{2}+4 \Delta x_{i}\left(F+F_{s v}\right)_{i}}}\right], \ldots
$$

where $\Delta x_{i}$ represents mesh sizes. The computations were performed on a 16-core parallel computer at the Data Processing Center of Kyoto University.

\section{Results and Discussion}

\subsection{Experimental Results}

We systematically varied the jet velocity, nozzle-to-plate distance, nozzle-to-nozzle spacing, and velocity of the water film and observed the images. Because the jet and film velocities considerably influence the flow structures of the twinjet impingement, we explain their effects. First, we studied the effects of varying the jet velocity on the flows. Figure 3 shows the experimental results obtained under the following conditions: liquid film velocity (velocity of the solid's motion) $V_{f}=V_{s}=0.4 \mathrm{~m} / \mathrm{s}$; nozzle-to-plate distance $H=40 \mathrm{~mm}$; and spacing between the two jet centers, $d_{n}=$ $30 \mathrm{~mm}$. The mean velocity at the pipe nozzle was varied as 

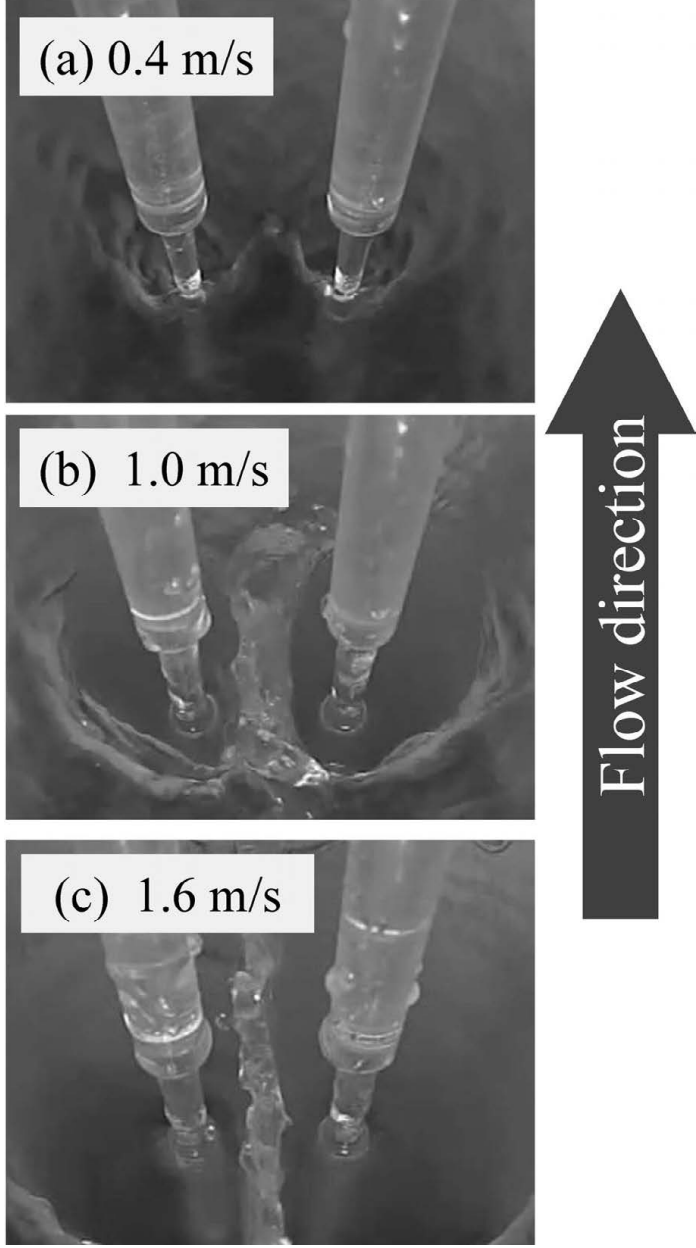

Fig. 3. Effect of varying jet velocity on flows when $V_{f}=V_{s}=$ $0.4 \mathrm{~m} / \mathrm{s}, H=40 \mathrm{~mm}, d_{n}=30 \mathrm{~mm}$, and $V_{0}=$ (a) 0.4 , (b) 1.0 , and (c) $1.6 \mathrm{~m} / \mathrm{s}$.

well: (a) $V_{0}=0.4 \mathrm{~m} / \mathrm{s}$, (b) $V_{0}=1.0 \mathrm{~m} / \mathrm{s}$, and (c) $V_{0}=1.6 \mathrm{~m} / \mathrm{s}$. In all cases, a pair of thin liquid film regions formed near the jet impact points; the areas of these regions grew as the jet velocity was increased. Owing to the flow film's inertia, the areas of the thin-film regions were small upstream of the jet impact points and large downstream. For (a), the thinfilm regions were present only near the impact point. There was little flow interaction between the two thin-film regions. The areas of the thin-film regions were larger under (b) than under (a). A couple of bow-shaped hydraulic jumps, where liquid thickness increased steeply, were generated on the upstream side. In addition, a fountain formed midway between the jets. The fountain under (c) was higher than that in the other two cases, and the areas of the thin-film regions were considerably larger.

Second, the effect of varying the liquid film's velocity on the flows was investigated under the conditions of $V_{0}=$ $0.8 \mathrm{~m} / \mathrm{s}, H=40 \mathrm{~mm}$, and $d_{n}=20 \mathrm{~mm}$. Figure 4 shows the results for the following film velocities: (a) $V_{f}=V_{s}=0.2 \mathrm{~m} / \mathrm{s}$, (b) $V_{f}=V_{s}=0.4 \mathrm{~m} / \mathrm{s}$, and (c) $V_{f}=V_{s}=0.8 \mathrm{~m} / \mathrm{s}$. Under (a), a couple of bow-shaped hydraulic jumps were located upstream of the jets' impact points. A pair of stable thin-film flow regions was formed. Under (b), thin-film regions were observed only downstream of the jets' impact points. Because the bow-shaped hydraulic jumps frequently impacted the vertical jets' cores, the flow motion near the

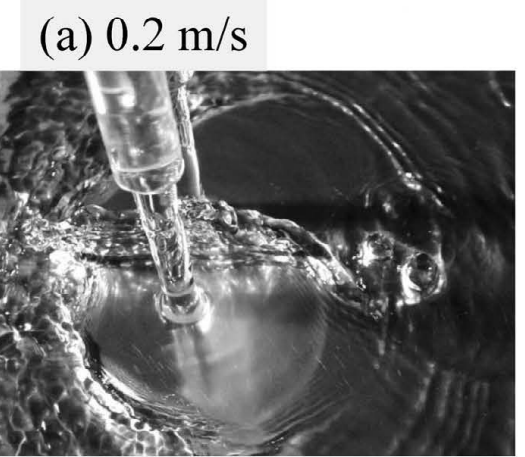

(b) $0.4 \mathrm{~m} / \mathrm{s}$

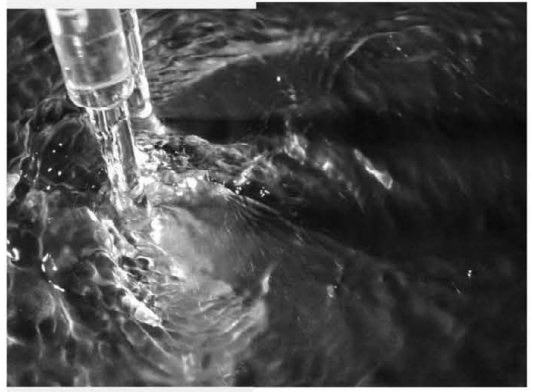

(c) $0.8 \mathrm{~m} / \mathrm{s}$

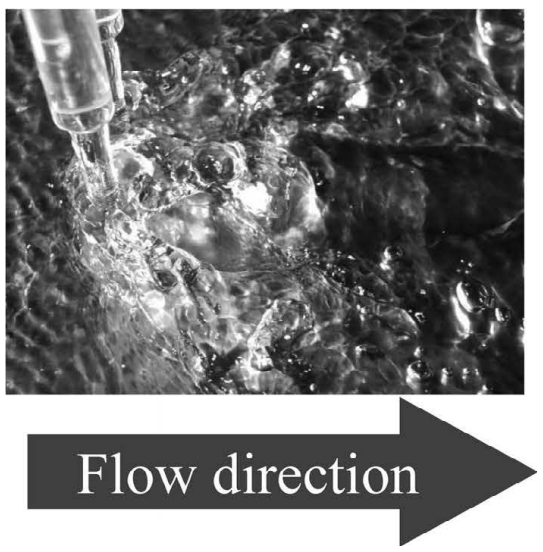

Fig. 4. Effect of varying film velocity on flows when $V_{0}=0.8 \mathrm{~m} / \mathrm{s}$, $H=40 \mathrm{~mm}, d_{n}=20 \mathrm{~mm}$, and $V_{f}=V_{s}=$ (a) 0.2 , (b) 0.4 , and (c) $0.8 \mathrm{~m} / \mathrm{s}$.

jet impact points was unsteady. Under (c), the bow-shaped hydraulic jumps strongly impacted the twin vertical jet cores. Consequently, the entire flow was very unstable. Furthermore, the fountain between the two jets fluctuated considerably.

We conducted experiments under various conditions and roughly categorized the flows into three modes based on the flow motions of the bow-shaped hydraulic jumps. For the first mode (shown in Fig. 4(a)), a couple of bow-shaped hydraulic jumps formed upstream of the jets' impact points. A pair of thin-film zones was observed not only downstream but also upstream of the jets' impact points. The flows were relatively stable near the jets' impact points. For the second mode (shown in Figs. 4(b) and 4(c)), the flow was unsteady because of interactions between the bow-shaped hydraulic jumps and the jet cores. For convenience, the first and second modes are called "stable mode" and "unstable mode," respectively. The third mode was a transition between the stable and unstable modes, but its definition is somewhat 
subjective. Figure 5 shows the flow modes under various experimental conditions. The unstable mode clearly appeared for large film-flow velocities $V_{f}$. The unstable mode also occurred under conditions of small jet velocities $V_{0}$ because the upstream thin-film regions disappeared for small jet velocities, as shown in Fig. 3(a). The critical boundary at which the stable mode was obtained apparently depended on $V_{0}, V_{f}, H$, and $d_{n}$.

Many prior studies ${ }^{18)}$ have examined circular jet cooling. In many studies, quasi-steady impinging jets were used where the thin-film region was stable and present. Some of the results obtained from these experiments can be applied to stable flows. Theoretical analysis is also possible near the impact point at least. In contrast, little prior data are available for unstable flows. Fully 3D numerical simulations and/ or thorough experiments are indispensable. In other words, the appropriate analysis method should be chosen depending on the flow mode. Thus, the critical boundary at which the stable mode is obtained needs to be predicted; this is explained in the next subsection.

\subsection{Critical Conditions of Stable Mode}

In our previous work, ${ }^{9)}$ a single jet impinging on a film flow over a moving solid was studied, and similar flow modes were explored. In addition, a simple theoretical model for predicting the critical boundary at which the stable mode is obtained was proposed based on a steady irrotational flow theory for inviscid fluids. In the present study, we attempted to build a similar model for twinjet impingement. First, we assumed that the thickness of the film flow issuing from the slot nozzle is comparable to the thickness of the radial flow formed by impingement of the circular jet. The following mean velocities integrated across the liquid film are introduced:

$$
\begin{aligned}
& \bar{u}(x, y)=\frac{1}{\delta} \int_{0}^{\delta} u(x, y, z) d z \\
& \bar{v}(x, y)=\frac{1}{\delta} \int_{0}^{\delta} v(x, y, z) d z \\
& \bar{w}(x, y)=\frac{1}{\delta} \int_{0}^{\delta} w(x, y, z) d z
\end{aligned}
$$

where $\delta$ is the local film thickness. The mean velocity component in the $z$-direction can be regarded as $\bar{w} \sim 0$ except in the regions of jet impact, because the liquid flow is almost parallel to the solid surface. Based on these model assumptions, the flow described by the mean velocities becomes 2D. Figure 6 shows flow modeling for the mean velocity profile $(\bar{u}, \bar{v})$. There are three flow components: a pair of radial flows formed because of twinjet impingement and a uniform flow issuing from the slot nozzle. Assuming that the flow is stable, inviscid, and irrotational, we have the following complex potential function: ${ }^{19)}$

$$
\begin{aligned}
F(x+i y)= & V_{f}(x+i y)+\frac{q}{2 \pi} \ln \left\{x+i\left(y+\frac{d_{n}}{2}\right)\right\} \\
& +\frac{q}{2 \pi} \ln \left\{x+i\left(y-\frac{d_{n}}{2}\right)\right\}
\end{aligned}
$$

The first term on the right-hand side represents a uniform flow velocity $V_{f}$ parallel to the $x$-axis. The second and third
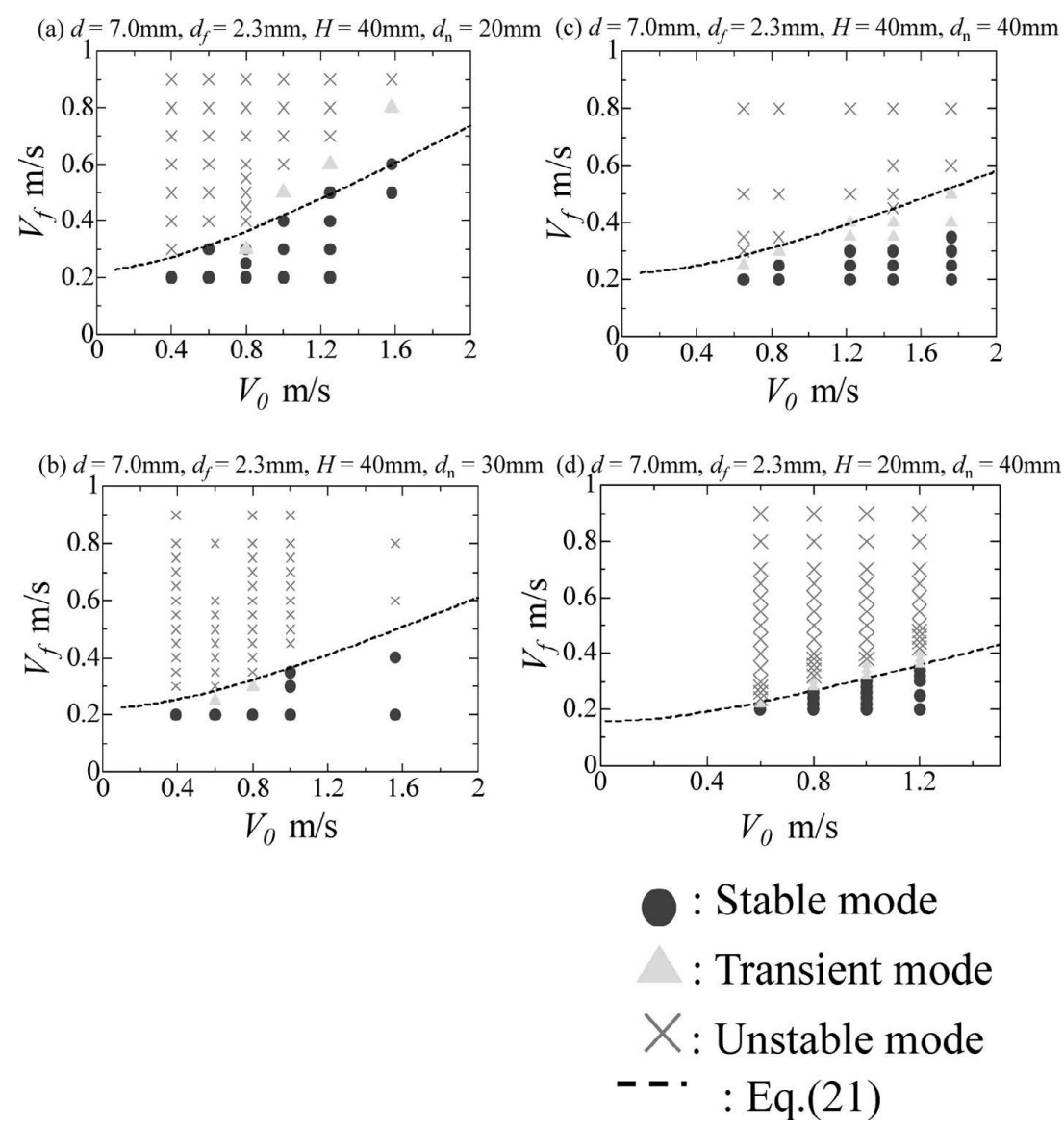

Fig. 5. Flow mode for various experimental conditions. 
terms represent point source terms that indicate that the fluid flows outward with strength $q$; this can be related to the radial jet velocity $V_{j}$ and jet radius $r_{j}$ at the impact point as follows:

$$
q=2 \pi r_{j} V_{j}
$$

The mean velocity components can be determined from the complex potential function as follows: ${ }^{18)}$

$$
\begin{aligned}
& \bar{u}=V_{f}+r_{j} V_{j}\left\{\frac{x}{x^{2}+\left(y+d_{n} / 2\right)^{2}}+\frac{x}{x^{2}+\left(y-d_{n} / 2\right)^{2}}\right\} \\
& \bar{v}=r_{j} V_{j}\left\{\frac{y+d_{n} / 2}{x^{2}+\left(y+d_{n} / 2\right)^{2}}+\frac{y-d_{n} / 2}{x^{2}+\left(y-d_{n} / 2\right)^{2}}\right\} \ldots \ldots .
\end{aligned}
$$

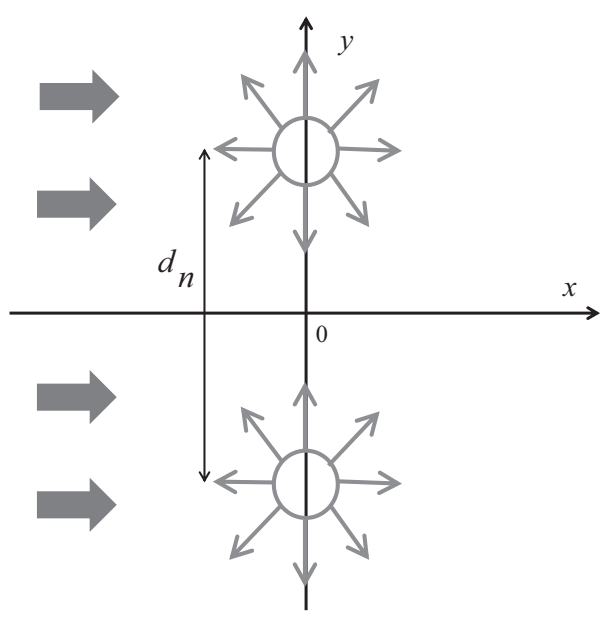

Fig. 6. Flow modeling.
By substituting $\bar{u}=\bar{v}=0$ in Eq. (16), we obtained the location of the stagnation points in the following form:

$$
(x, y)=\left\{\begin{array}{l}
\left(-\frac{r_{j} V_{j}}{V_{f}}, \pm \sqrt{\left(\frac{d_{n}}{2}\right)^{2}-\left(\frac{r_{j} V_{j}}{V_{f}}\right)^{2}}\right) \quad V_{f}^{2} \geq \frac{4\left(r_{j} V_{j}\right)^{2}}{d_{n}^{2}} \\
\left(-\frac{r_{j} V_{j}}{V_{f}} \pm \sqrt{\left(\frac{r_{j} V_{j}}{V_{f}}\right)^{2}-\left(\frac{d_{n}}{2}\right)^{2}}, 0\right) \quad V_{f}^{2}<\frac{4\left(r_{j} V_{j}\right)^{2}}{d_{n}^{2}}
\end{array}\right.
$$

Equation (17) suggests that depending on the flow conditions, a maximum of two stagnation points exist. In the present experiments, the flow was always in the stable mode under $V_{f}^{2}<4\left(r_{j} V_{j}\right)^{2} / d_{n}{ }^{2}$. Hence, the stagnation points for $V_{f}^{2} \geq 4\left(r_{j} V_{j}\right)^{2} / d_{n}^{2}$ are important in terms of predicting the critical conditions.

In our previous study, ${ }^{9)}$ the flows were stable when the distance of the stagnation point $(\bar{u}=\bar{v}=0)$ from the jet impact point was roughly four times the jet radius $r_{j}$. Although this finding was not made using twinjet impingement, we applied the relationship to the present theoretical model. Therefore, we have

$$
\sqrt{\frac{d_{n}^{2}}{2}-d_{n} \sqrt{\left(\frac{d_{n}}{2}\right)^{2}-\left(\frac{r_{j} V_{j}}{V_{f}}\right)^{2}}} \geq 4 r_{j}
$$

Here, the left-hand side of the equation represents the distance between a stagnation point $(\bar{u}=\bar{v}=0)$ and a jet impact point.

According to Bernoulli's equation and the continuity
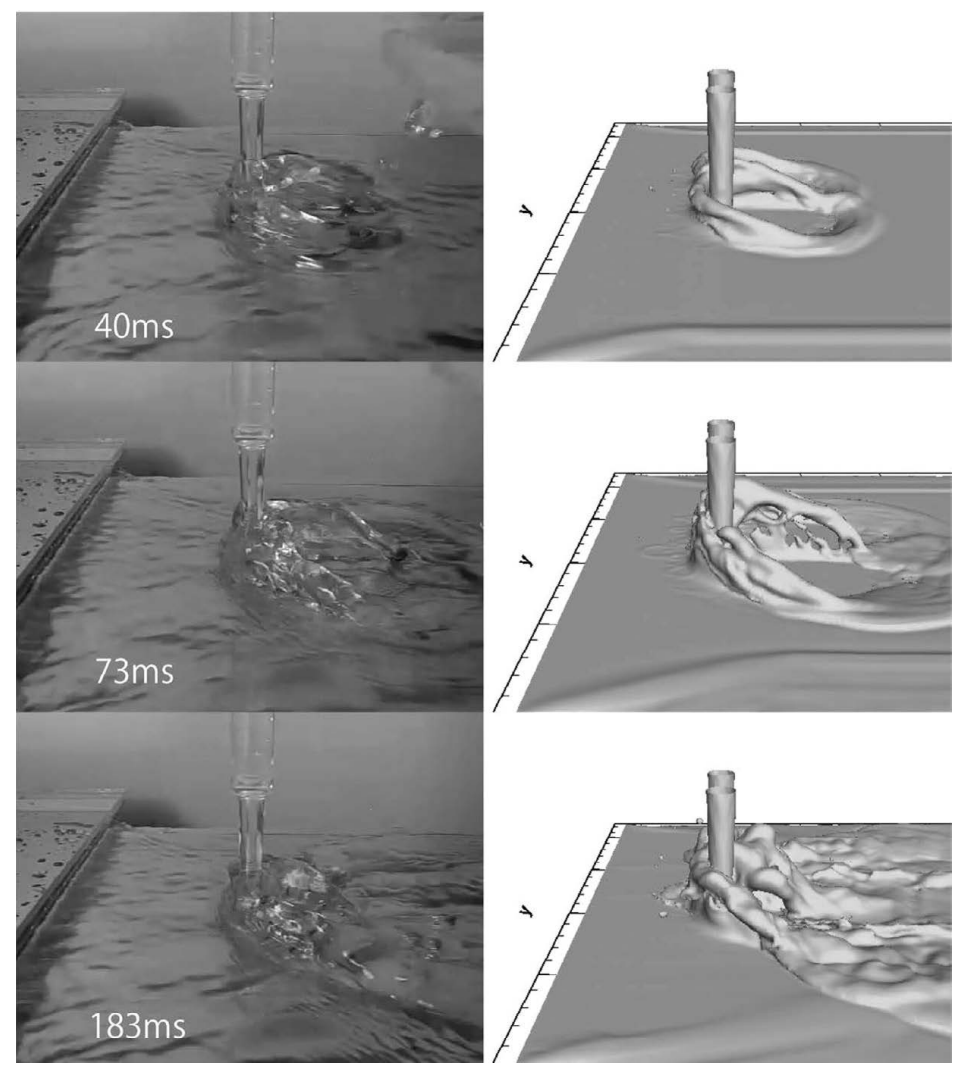

Fig. 7. Comparison of numerical and experimental results. 
equation, we have the following relations:

$$
\begin{aligned}
& V_{j}=\sqrt{V_{0}^{2}+2 g H}=V_{0} \sqrt{1+2 / F r_{H}^{2}}, \quad F r_{H}=V_{0} / \sqrt{g H} \ldots \\
& r_{j}=d / 2 \sqrt{V_{0} / V_{j}}
\end{aligned}
$$

where $g$ and $\mathrm{Fr}_{H}$ denote the gravitational acceleration and Froude number, respectively. By substituting Eqs. (19) and (20) into Eq. (18), we obtain the following equation as a function of three dimensionless parameters:

$$
\left(\frac{V_{0}}{V_{f}}\right)^{2}=16\left\{\left(1+\frac{2}{F r_{H}^{2}}\right)^{-1}-4\left(\frac{d}{d_{n}}\right)^{2}\left(1+\frac{2}{F r_{H}^{2}}\right)^{-\frac{3}{2}}\right\} .
$$

As shown in Fig. 6, the predicted critical boundaries given by Eq. (21) agree reasonably well with those in the experiments; however, the model's assumptions are oversimplified compared to the actual flows. Further consideration is needed for establishing a more useful correlation capable of predicting the critical boundary. This is a challenge for future work.

\subsection{Numerical Simulation}

As noted previously, twinjet impingement on a moving liquid film produces three flow modes. In the stable mode, the flows are almost steady in the thin-film regions formed around the jet impact points. As discussed in previous subsections, predicting the flow structures theoretically should be possible. However, the flows categorized as unstable are essentially unsteady, and numerical simulations are very useful to understanding their physics. Therefore, we discuss unstable mode flows in this subsection.

First, the numerical model used here was validated by comparing its numerical results with experimental results. A transient flow motion that formed shortly after initial contact of the twinjet with the liquid film was investigated under the following conditions: $V_{0}=0.8 \mathrm{~m} / \mathrm{s}, V_{f}=V_{s}=$ $0.8 \mathrm{~m} / \mathrm{s}, H=40.0 \mathrm{~mm}$, and $d_{n}=20 \mathrm{~mm}$. These conditions were the same as those shown in Fig. 4(c). Figure 7 compares the numerical results with those of high-speed video-
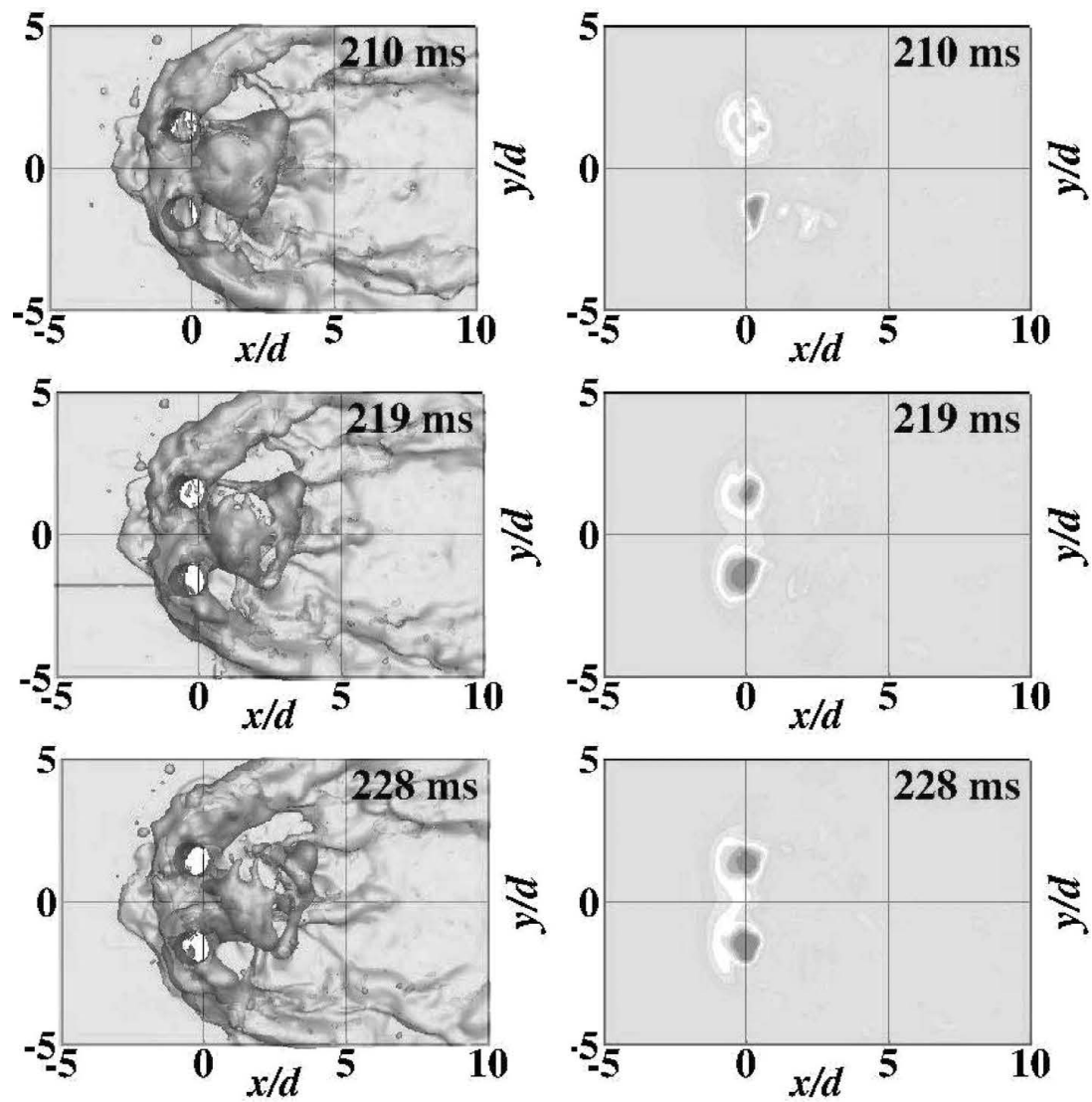

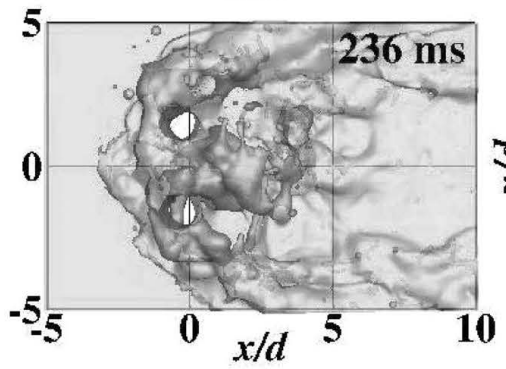

(a)

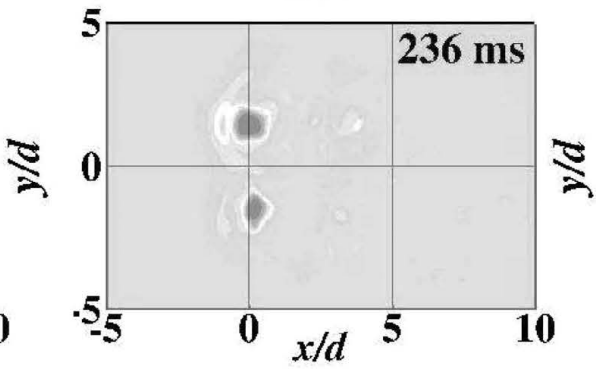

(b)

$-300-100) 100 \quad 300 \quad 500$

Fig. 8. Time evolution of (a) free surface and (b) pressure contour at solid surface. Note that the flow conditions are the same as those in Fig. 4(c). 
graphy. The time elapsed after the twin liquid jets touch the liquid film is shown in each image. The initial contact of the twinjet with the flowing liquid film was realized experimentally as follows. Initially, a baffle plate was set between the pipe nozzles and the moving plate to prevent direct impingement of the twinjet on the liquid film. The plate was then quickly removed so that the twinjets impinged on the liquid film. Because the leading edge of the falling twinjet was somewhat distorted and high-speed videography was carried out at $300 \mathrm{fps}$, the deviation in elapsed time could be a maximum of $4 \mathrm{~ms}$.

Immediately after the twinjet impinged on the film flow, two hydraulic jumps were formed from the impact points. Owing to the inertia of the liquid film flow, the hydraulic jumps spread mainly in the downstream direction; this was followed by the formation of a pair of thin-film regions. A fountain was also observed at the midpoint between the two jets. The heights of these hydraulic jumps and fountain increased over time, peaked, and then decreased because of gravity. The free surface on the downstream side was very wavy at $183 \mathrm{~ms}$. The predicted free surface motion agreed moderately well with the experimentally obtained results.

High-speed videography revealed that a transient flow motion occurred approximately $200 \mathrm{~ms}$ after the initial contact between the twinjet and the film flow. Thereafter, the flow motion was ordinary, as shown in Fig. 4(c). Figure 8 shows (a) the top view of the free liquid surface and (b) the pressure contour at the solid surface after $200 \mathrm{~ms}$. The free surface was very wavy and similar to the experimental results shown in Fig. 4(c). The pressure was high near the impact point and was time-variant. The pressure profile was asymmetric along the midline between the two jets.

This unstable pressure behavior was ascribed to the contact between the hydraulic jump and the jet cores. Figure 9 depicts the time evolution of the pressure contour, velocity profile, and lines showing the free surface on a plane of $y=$ $10 \mathrm{~mm}$. As shown in all of the figures, the hydraulic jump directly touched the jet core. There was an air gap between the hydraulic jump and the jet core. The shape of the hydraulic jump's free surface varied considerably with time. On the downstream side, the liquid film was very thin. At 210 and $219 \mathrm{~ms}$, a small amount of air was present near the impact point, and the pressure near the jet impact point was lower than those at 228 and $236 \mathrm{~ms}$. At $236 \mathrm{~ms}$, a relatively high pressure region was seen upstream of the jet impact point $(x / d \sim-1.0)$, where a uniform liquid flow going downstream and a radially spreading flow formed because of the jet impact. A vertical upward flow also originated there and resulted in the formation of a hydraulic jump.

Figure 10 shows the numerical results for the following conditions: $V_{0}=0.8 \mathrm{~m} / \mathrm{s}, V_{f}=V_{s}=0.4 \mathrm{~m} / \mathrm{s}, H=40.0 \mathrm{~mm}$, and $d_{n}=20 \mathrm{~mm}$. These conditions were identical to those in the experiment shown in Fig. 4(b). Figure 5(a) shows that the flow conditions were close to the critical boundary. The predictions agreed reasonably well with the experimental results shown in Fig. 4(b). The bow-shaped hydraulic jumps touched the twinjet cores directly, and there was no thin-film region upstream of the jet impact points. The shape of the free liquid surface varied with time. Because the interaction of the bow-shaped hydraulic jump with the jet cores was milder than that shown in Fig. 8, the pressure was stable and
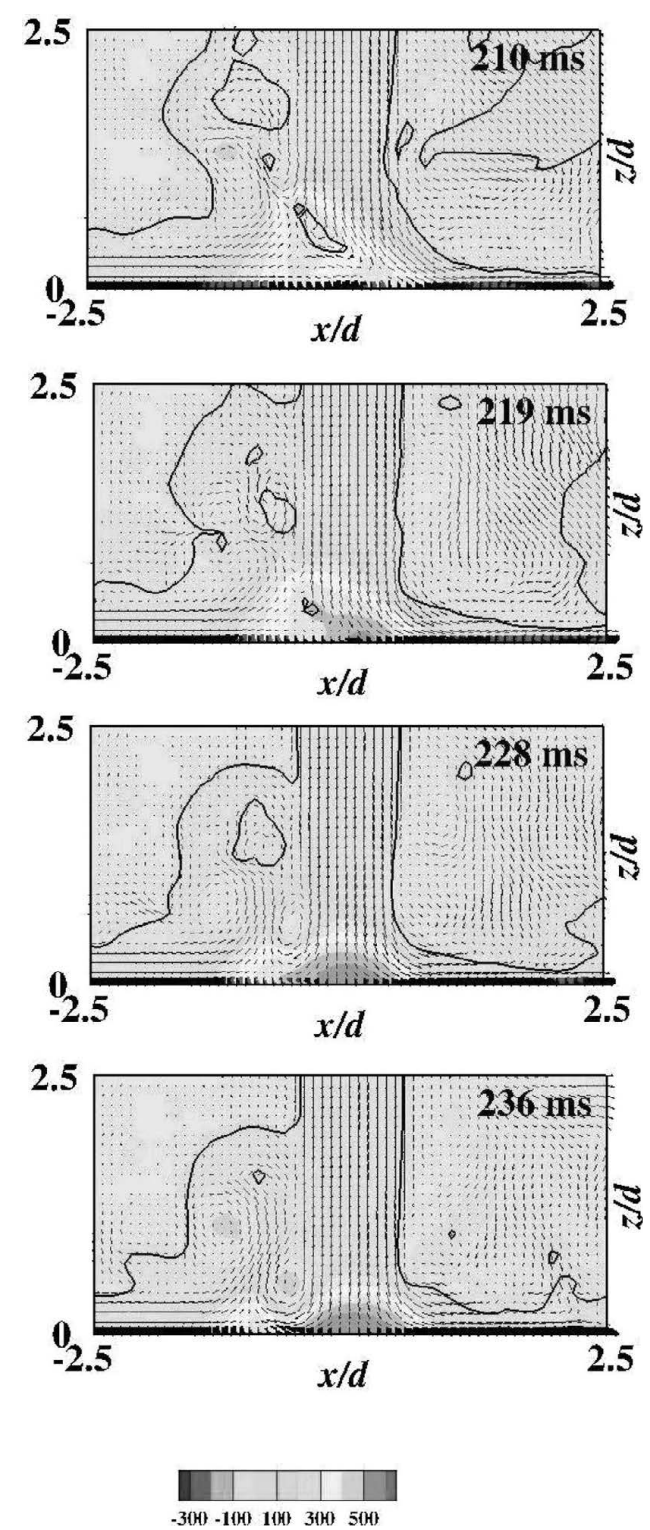

Fig. 9. Pressure contour and velocity profile for vertical plane of $y=10 \mathrm{~mm}$.

high at the jet impact points. Moreover, a slightly high pressure area was observed at the fountain between the two jets.

\section{Conclusion}

The flow properties of twin circular water jets impinging on a moving surface covered with a water film were investigated through experiments and 3D computer simulations. The main results obtained in this study are summarized below.

(1) Three flow modes were observed. In the stable mode, a pair of thin-film regions formed near the jet impact points; this was followed by a couple of bow-shaped hydraulic jumps and a fountain between the two jets. With decreasing jet impingement velocity, or increasing film velocity, the bow-shaped hydraulic jumps shifted toward the vertical jet cores. The flow changed to the transient mode and, subsequently, to the unstable mode. In the unstable mode, the hydraulic jumps impacted the jet cores so frequently that the flow was essentially unsteady. 

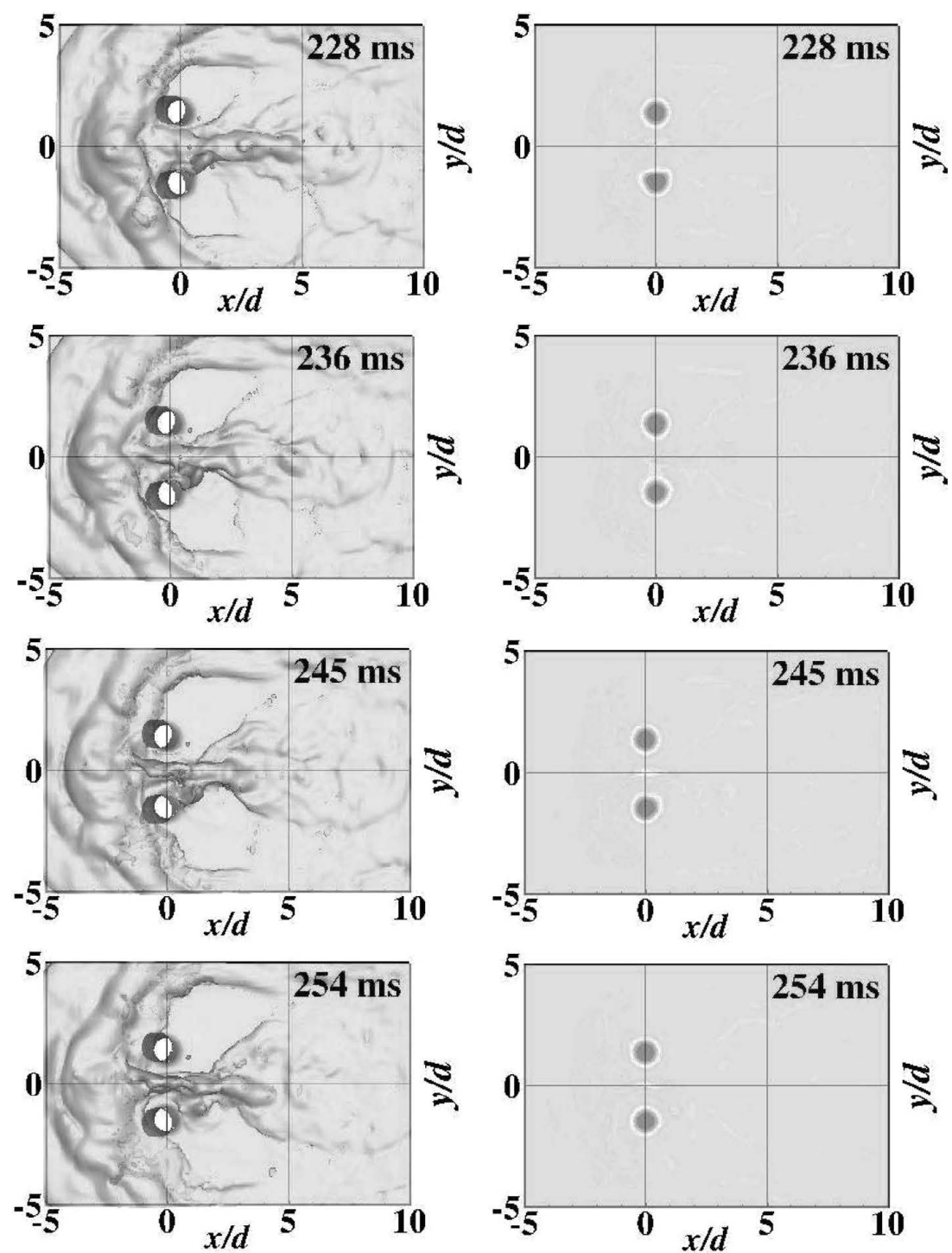

(a)

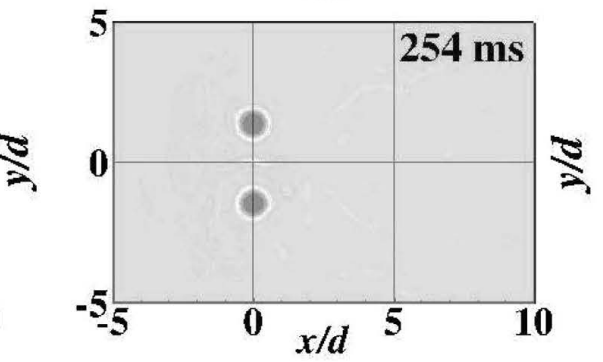

(b)

Fig. 10. Time evolution of (a) free surface and (b) pressure contour at solid surface. Note that the flow conditions are the same as those in Fig. 4(b).

(2) A simple theoretical model for predicting the critical boundary at which the stable mode is obtained was built based on a steady irrotational flow theory for inviscid fluids. The predictions of this model were found to agree reasonably well with the experimental results.

(3) Twinjet impingement for the unstable mode was investigated through numerical simulations. The predictions agreed reasonably well with the experimental results. The pressure profile near the jet impact points varied with time when the bow-shaped hydraulic jumps strongly impacted the jet cores.

\section{REFERENCES}

1) S.-J. Chen and A. A. Tseng: Int. J. Heat Fluid Flow, 13 (1992), 358.

2) N. Hatta, Y. Tanaka, H. Takuda and J. Kokado: ISIJ Int., 29 (1989), 673.

3) N. Hatta and H. Osakabe: ISIJ Int., 29 (1989), 919.

4) D. A. Zumbrunnen, F. P. Incropera and R. Viskanta: Exp. Therm.
Fluid Sci, 3 (1990), 202.

5) M. J. Cho, B. G. Thomas and P. J. Lee: Metall. Mater. Trans. B, 39 (2008), 593.

6) I. S. Park: ISIJ Int., 51 (2011), 743.

7) I. S. Park: ISIJ Int., 51 (2011), 1864.

8) I. S. Park: ISIJ Int., 52 (2012), 1080.

9) H. Fujimoto, Y. Suzuki, T. Hama and H. Takuda: ISIJ Int., 51 (2011), 1497.

10) S. Ishigai, S. Nakanishi, M. Mizuno and T. Imamura: Bull. JSME, 20 (1977), 85

11) R. P. Kate, P. K. Das and S. Chakraborty: J. Fluid Mech., 590 (2007), 355.

12) D. Gueyffier, J. Li, A. Nadim, R. Scardovelli and S. Zaleski: $J$. Comput. Phys., 152 (1999), 423.

13) R. Scardovelli and S. Zaleski: J. Comput. Phys., 164 (2000), 228.

14) C. W. Hirt and B. D. Nichols: J. Comput. Phys., 39 (1981), 201.

15) M. Sussman and E. G. Puckett: J. Comput. Phys., 162 (2000), 301.

16) J. U. Brackbill, D. B. Kothe and C. Zemach: J. Comput. Phys., 100 (1992), 335.

17) T. Yabe, F. Xiao and T. Utsumi: J. Comput. Phys., 169 (2001), 556.

18) B. W. Webb and C.-F. Ma: Adv. Heat Transfer, 26 (1995), 105.

19) H. Lamb: Hydrodynamics, 6th ed., Cambridge University Press, London, (1932), 62. 